\title{
Kernos
}

Revue internationale et pluridisciplinaire de religion grecque antique

$10 \mid 1997$

Varia

\section{Ph. DAIN, Mythographe du Vatican I}

\section{Bruno Rochette}

\section{(2) OpenEdition \\ Journals}

\section{Édition électronique}

URL : http://journals.openedition.org/kernos/680

DOI : $10.4000 /$ kernos. 680

ISSN : 2034-7871

Éditeur

Centre international d'étude de la religion grecque antique

Édition imprimée

Date de publication : 1 janvier 1997

Pagination : 340-343

ISSN : 0776-3824

Référence électronique

Bruno Rochette, «Ph. DAIN, Mythographe du Vatican I », Kernos [En ligne], 10 | 1997, mis en ligne le 12 avril 2011, consulté le 22 septembre 2020. URL : http://journals.openedition.org/kernos/680 ; DOI : https://doi.org/10.4000/kernos.680 
d'une nouvelle renaissance qui l'accompagne, importés directement d'Italie. Dans une seconde partie, l'A. interroge de nombreux textes du Moyen âge pour connaître le sort des vestiges antiques contemporains conservés dans les diverses régions de France. Ces textes révèlent l'attrait des gens d'alors pour les ruines (temples, amphithéâtres, aqueducs, murs des villes), les sculptures (sarcophages, statues, cippes) et les objets d'art antiques qui les entourent. Ils dévoilent aussi les raisons de cet engouement et les variations d'intensité de cet intérêt depuis l'époque de Grégoire de Tours jusqu'à celle de Jean Fouquet. Dans une troisième et dernière partie, l'A. présente enfin les influences antiques dans l'art du Moyen âge en les rapportant à leur source. La moisson est importante pour les périodes carolingienne et romane, mais très faible pour l'époque gothique, au début de laquelle on remarque pourtant quelques efforts isolés et sans lendemain, ceux du "Maître des Figures Antiques " et de son élève, Villar de Honnecourt.

Les quelques coquilles qui subsistent dans le texte témoignent de l'absence de remise à jour. Le travail n'en souffre pas : l'argumentation reste convaincante et exemplaire. Il a ouvert la voie à des recherches plus ciblées dont les résultats sont présentés lors d'expositions qui font date : pour la renaissance carolingienne, Karl der Grosse (Aix-la-Chapelle, 1965), pour les efforts isolés à l'aube du gothique, The Year 1200 (New York, 1970), pour l'humanisme du $\mathrm{xv}^{\mathrm{e}}$ siècle, Les fastes du gotbique (Paris, 1980). Il convient aussi de mentionner les recherches d'E. Panofsky, Renaissance and Renascences in Western Art, publiées en 1960; il s'interroge sur l'opportunité de distinguer la Renaissance, phénomène unique, et les renouveaux médiévaux qui constitueraient autant de renaissances.

Isabelle LECOCQ

(Université de Liège)

Philippe DaIN, Mythographe du Vatican I. Traduction et commentaire, Besançon-Paris, 1995. 1 vol. 15, $5 \times 24 \mathrm{~cm}$, XIX+244 p. (Annales Littéraires de l'Université de Basançon, 579. Centre de Recherches d'Histoire Ancienne, 151. Institut Félix Gaffiot, 12. Lire les polythéismes, 5). ISBN : 2-251-60579-7.

Nul doute que, dans le domaine francophone, 1995 pourra être considéré comme une année faste pour le Premier Mythograpbe du Vatican, puisque deux traductions françaises de cet ouvrage curieux ont vu le jour presque simultanément. L'une, accompagnée du texte latin, établi par N. ZORZETTI, est due à J. BERLIOZ et a paru dans la Collection des Universités de France. L'autre, sans le texte latin, est l'ouvre de Ph. DAIN et est publiée dans une collection diffusée aussi par la Maison d'éditions « Les Belles Lettres ». Les spécialistes, mais aussi les lecteurs moins versés dans le domaine de la mythologie gréco-romaine, peuvent se réjouir d'autant plus que les deux traductions sont accompagnées d'abondantes notes destinées à fournir toutes les explications utiles à l'intelligence d'un texte rempli d'allusions.

C'est en 1831 qu'Angelo MaI publia, sous l'appellation de Mytbograpbi Vaticani, les textes de trois auteurs dont il venait de découvrir les manuscrits à la Bibliothèque Vaticane. Ces traités de mythologie offrent un choix de mythes gréco-latins, repris à des mythographes antérieurs, comme Fulgence, auxquels sont venus se greffer des récits issus de la tradition romaine. Nommés Mythographes I, II et III d'après l'ordre chronologique supposé d'âge ou de publication, - les deux premiers écrivains semblent 
avoir vécu au $\mathrm{X}^{\mathrm{e}}$ siècle, le troisième au $\mathrm{XII}^{\mathrm{e}}$, - ces auteurs anonymes firent l'objet d'éditions successives, qui améliorèrent celle de MAI, où les erreurs et les omissions étaient légion. Toutefois, aucune traduction en français n'en avait encore été réalisée. Celle qui est présentée ici est certainement méritoire, non seulement parce qu'elle est la première, mais parce qu'elle rend un texte difficile, au style haché, véritable « collage » à certains moments, où foisonnent les discordances temporelles et modales.

L'introduction traite de l'identité de l'auteur, sans doute un clerc, de ses qualités d'écrivain, qui sont assez médiocres, de la nature de l'œuvre - sources et disposition des légendes - et enfin de sa finalité. Destiné à être lu dans les écoles cléricales, le recueil a une dimension religieuse incontestable. Les remaniements que l'A. a opérés dans la présentation de certains mythes sont destinés à leur donner une coloration chrétienne. Toutefois, si cette orientation de l'œuvre ne peut être contestée, les exemples présentés par l'A. à la page XIII ne me paraissent pas tous convaincants. De plus certains développements de cette page sont très elliptiques, parfois même sibyllins (p. ex. « certes, le texte est emprunté à Isidore de Séville, mais le mythographe connaît. bien Isidore, ce qui, sur le plan de sa 'chrétienté', revient au même »).

La traduction demande peu de commentaires. Si elle est quelquefois lourde, c'est parce que l'original latin est lourd. Si elle est à certains endroits sinueuse, c'est parce que le style du mythographe l'est aussi. Souvent embarrassé par le style déroutant du mythographe, l'A. tente de rendre au mieux les effets du texte latin, mais ces efforts se font parfois au détriment de l'exactitude, comme il le reconnaît lui-même. Tout traducteur a fait l'expérience du non possum. En revanche, le commentaire, où l'A. dit " avoir collationné les recherches les plus récentes " (p. 230), appelle de nombreuses réserves. Sa conception même ne me paraît pas correspondre à ce qu'un lecteur est en droit d'attendre d'une traduction annotée. Si des notes sont nécessaires « à plusieurs niveaux $»$ ( $\mathrm{p}$. XVIII), celles qui sont proposées comportent à la fois trop et trop peu. Trop, parce qu'elles contiennent des remarques superflues sur la manière dont le texte a été traduit (p. ex. p. 69, n. 1 « c'est la traduction du texte, tel qu'il se présente. Mais ce texte demande des explications », p. 76, n. 3: "il ne s'agit pas, dans le cadre de cette étude, .... Je renvoie pour ceci à... » ou encore p. 87, n. 1 « cette première phrase traduite telle qu'elle se présentait - appelle quelques remarques $»)$. De telles phrases « introductives » sont inutiles et alourdissent considérablement le commentaire, qui devrait aller droit au but en se limitant à l'essentiel. Trop encore, parce que certaines indications auraient aisément pu être omises ou, à tout le moins, exprimées de façon plus concise. Il est inutile, par exemple, de citer si souvent le Dictionnaire de la mythologie grecque et romaine, Paris, $1963^{3}$, de P. GRIMAL ${ }^{34}$, mentionné comme une "Bible » de la mythologie, alors qu'il n'est pas exempt de méprises. Tout aussi inutiles sont la plupart des renvois aux articles du Dictionnaire... de DAREMBERG et SAGLIO, que l'A. cite à tout propos et hors de propos. Trop peu, parce que certaines références aux textes anciens méritaient droit de cité et que les mentions récurrentes d'ouvrages généraux, voire de manuels élémentaires ou didactiques ${ }^{35}$, auraient dû faire place à un renvoi précis à une étude spécialisée. Je me contenterai de relever les exemples qui m'ont paru les plus frappants.

34 Il faudrait de toute façon citer une édition plus récente. Il y a une dixième édition de 1990.

35 P. ex. P. LÉVÊQUE, L'aventure grecque, Paris, 1964, et S. SAÏD, Approches de la mythologie grecque, Paris, 1994 (Coll. '128'). 
P. 1-3, à propos du mythe de Prométhée, aucune référence à la tragédie d'Eschyle, ni au petit livre de L. SÉCHAN, Le mythe de Prométbée, Paris, 1951 (Mythes et religions, 28). P. 3, n. 4 : à propos de la valeur symbolique de cor; les travaux d'A. HERMANN, Das steinharte Herz, in JbAC, 4 (1961), p. 77 (la « philosophie du cœur ») et 88-89 et d'A. GUILLAUMONT, Le sens des noms du cour dans l'antiquité, in Études carmélitaines, 29 (1950), p. 41-81 fournissent des renseignements beaucoup plus utiles que les ouvrages lexicographiques cités. P. 16, n. 1: le sacrifice humain a été récemment étudié par P. BONNEchere (Kernos Supplément, 4 [1994]). P. 17 : pour la légende d'Io, pas de référence au Prométhée enchaîné d'Eschyle. De même, à propos d'Iphigénie, pas de renvoi aux tragédies d'Euripide. P. 28-29 : sur la légende de Cléobis et Biton, aucune allusion à Hérodote. P. 30$31, \mathrm{n}, 4$ : à propos de Roma et Romulus, le problème est en fait beaucoup plus complexe ${ }^{36}$. Il aurait fallu citer au moins Ennius $\left(55 \mathrm{~V}^{3}=47\right.$ Skutsch v. 6 : certabant Urbem Romam Remoramne uocarent). À l'ouvrage de W. SCHULze, Zur Geschichte lateiniscber Eigennamen, - qui date de 1904, non de 1966 -, des références plus récentes auraient dû être ajoutées, p. ex. E. JUNG, Les noms du. Tibre et de Rome, in RIO, 24 (1972), spéc. p. 39-61 et H. SOLIN, Varia onomastica IV: Gibt es einen Franennamen Roma?, in ZPE, 39 (1980), p. 249-254. P. 44, n. 7 : à propos de Fimbria, cognomen de la gens Flawia, citer I. KAJANTO, The Latin Cognomina, Helsinki, 1965 [Rome, 1982], p. 223. P. 66, n. 4 : était-il bien utile de consacrer une longue note aux causes de la blessure de Philoctète et de mentionner l'essai d'identification du serpent qui mordit le héros ? Le détail est anecdotique ( $c f$. de toute façon, M. DELCOURT, Stérilités mystérieuses et naissances maléfiques dans l'antiquité classique, Liège, 1938 [Paris, 1986], p. 105-108). P. 83, n. 2 : est-il nécessaire, pour comprendre le texte du mythographe, de dire que « les versions rapportant la descente aux Enfers d'Orphée et sa mort sont nombreuses " et que "la plus pathétique me semble être celle de Virgile »? Un jugement subjectif n'a pas sa place dans un commentaire mythologique. P. 84, n. 1: à propos de la Lune, citer l'étude de Cl. PrÉAuX, La line dans la pensée grecque, Bruxelles, 1973. P. 87, n. 1 : la remarque fait double emploi avec le (sic) inséré dans la traduction. Ibid., n. 2 : la phrase qui justifie la traduction n'est compréhensible que lorsqu'on a le texte latin sous les yeux. P. 95-96: pas de référence à Euripide pour la légende d'Alceste. P. 107, n. 4 : est-il nécessaire de rappeler l'étymologie de Jupiter ? P. 12, n. 4 : faut-il vraiment consacrer une note à la nature et au rôle des Fétiaux, surtout en citant, encore et toujours, un article du DAREMBERG et SAGLIO ? P. 155, n. 2 : à propos de la terminologie botanique, on attendrait un renvoi au lexique de J. ANDRÉ. P. 159, n. 1 : peut-on être certain que c'est Lactance qui reprend Servius et non l'inverse ? P. 165, n. 1 : la longue justification de la traduction $(9$ lignes) est-elle nécessaire? P. 184, n. 3 : le nom du Tibre a été étudié par E. JUNG (cf. supra). P. 166, n. 3 : à propos du problème textuel de la conclusion de la Quatrième Églogue (v. 60-63), l'interprétation de J. CARCOPINO, qualifiée d'originale par l'A., est loin d'être assurée. Là aussi, le problème est beaucoup plus complexe ${ }^{37}$ et n'intéresse qu'indirectement le texte du mythographe. P. 185, n. $4:$ à propos d'Euphorion de Chalcis, qui inspira Gallus, est-il raisonnable de citer l'ouvrage de F. PLESS1S qui date de 1903 ? P. 194, n. 13 : l'identification proposée par J. CARCOPINO - sans précision bibliographique - entre le Numicus, petit fleuve parallèle au Tibre, avec le Canale dello Stagno est une simple hypothèse. Une vérification dans un ouvrage récent sur la topographie romaine s'impose (L. RICHARDSON et E.M. STEINBY). P. 195, n. 16 : est-il nécessaire de citer, pour le terme Laurolauinium, le Dictionnaire de GAFFIOT, qui reproduit le texte de Servius ? P. 196, n. 2 : à propos de l'authenticité du Rhésos, est-il utile de reproduire trois mots du Que sais-je? de S. SAÏD, lorsqu'il existe tout un livre consacré à ce problème? - D'autres défauts viennent encore s'ajouter à ces remarques. La présentation typographique comporte des aberrations : les parenthèse ouvertes et pas fermées et inversement, les références bizarrement placées, les alinéas dans les notes, l'accentuation du grec quelquefois erronée ( $c f$. p. 3) ou absente ( $c f$. p. 155), l'emploi d'un double ? ou !, la mention op. cit. qui ne paraît renvoyer à rien, les imprécisions dans certaines références aux auteurs anciens (p. ex. p. 73, n. 4 : Phérécyde, cité par Athénée, livre XI) ou à la littérature secondaire (p. ex. p. 31, n. 4), l'emploi d'une terminologie un peu déroutante ( $c f$. p. ex. p. 31, n. 5 le « comparatiste » entre guillemets pour parler de G. DUMÉzIL ou encore « fautes de copie » p. 154, n. 1), les incohérences dans l'ars citandi (p. ex. p. 177, n. 2 et p. 178, n. 5), les abréviations curieuses, l'emploi des grandes

36 Cf. ma note 'Póp $\mu \dot{\rho} \omega \dot{\mu} \eta$, à paraître dans Latomus.

37 R.D. WiLLIAMS, Virgil Eclogues 4.60-63, in CPb, 71 (1976), p. 119-121. 
capitales pour les titres d'ouvrages (p. 230), la lourdeur des mentions bibliographiques. Un exemple éclairant se trouve p. 106, n. 4 « on peut lire dans le Dict. des Antiq. grecques et romaines de DAREMBERG et SAGLIO, extrait de l'article de F. LENORMANT : '...' op. cit., T. I, $1^{\mathrm{e}}$ part., s.v. Baetylia p. 644a). " En outre, des indications telles que «le $12^{\mathrm{e}}$ tirage de l'édition Villeneuve aux Belles Lettres, tirage revu et corrigé par J. Hellegouarc'h en 1990 » (p. 63, n. 3) ou « la note d'A. Bellesort dans son édition de l'Énéide de Virgile, aux Belles Lettres, à propos du vers 54 du chant viII " (p. 76, n. 3) sont longues et inutiles. À côte de cette volonté de citer le dernier état d'une publication, l'A. recourt à des éditions anciennes (p. ex. p. 105, n. 4 : Photios, cité uniquement d'après l'édition de BEKKER). Enfin, la bibliographie finale manque d'ordre et de précision. On s'étonnera notamment de trouver Ennius cité d'après le Recueil de textes latins arcbaïques de ERNOUT - dont il faut citer la nouvelle édition de 1973, non celle de 1957 -, alors qu'il convient de se référer à la troisième édition de VAHLEN ou à celle de SKUTSCH (Oxford, 1985). De même, la section « Manuels, dictionnaires et encyclopédies » comporte trop d'ouvrages, dont certains ne semblent pas avoir été exploités dans le commentaire. Un tri eût certainement été nécessaire, ainsi qu'une toilette typographique. Dans cette liste, on trouve l'ouvrage ancien de GRUPPE (Munich, 1906), certes encore utile, mais on cherche en vain celui de M.P. NILSSON (Munich, 1967-19733) dans la même série du Handbuch der Altertumswissenscbaft. Les ouvrages de DETIENNE et de DUMÉZIL cités dans cette subdivision sont-ils bien à leur place ? On eût aimé aussi connaître le nom du ou des auteurs de la concordance aux Mythographi Vaticani I et II (Turnhout, 1987), présentée simplement comme émanant de l'Université catholique de Louvain (en grandes capitales, comme les noms d'auteurs). Pour les « petits commentateurs " de Virgile, cités p. 230, on doit à présent citer le recueil de I. BARABINO, A.V. NAZZARO et A. SCIVOLETTO (éds), Interpretationes Vergilianae Minores, I, Gênes, 1991 (Pubbl. del dipart. di arcb., fil. cl. e l. trad., n.s. 137).

Ces remarques n'ont pas pour but de dévaloriser le travail, méritoire, d'un traducteur, qui est de surcroît un pionnier. Elles voudraient seulement attirer l'attention sur la nécessité de fournir avec une traduction les éléments nécessaires à l'intelligence du texte, tous ces éléments, certes, mais seulement ces éléments. Une note précise et concise vaut mieux qu'un fatras d'érudition qui égare le lecteur dans un dédale de propos superflus, sans lui apporter, en définitive, la précision qui l'aurait éclairé. Ajoutez, quelquefois, et souvent effacez, disait Boileau.

Bruno ROCHETTE (Université de Liège)

\author{
Jesús-María Nieto IBÁÑez (coord.), Estudios de religión y mito en \\ Grecia y Roma. X Jornadas de Filología Clásica de Castilla y León, León, \\ Universidad, Secretariado de Publicaciones, 1995. 1 vol. $17 \times 24 \mathrm{~cm}, 295$ p., ill. \\ ISBN : 84-7719-517-X
}

Les royaumes de Castille et León ont occupé le centre géographique et culturel de l'Espagne, surtout pendant le Moyen âge et la Renaissance, grâce à leur histoire singulière, comme on le voit par l'existence de deux des Universités les plus anciennes d'Europe, celles de Salamanca et de Valladolid, auxquelles on a récemment ajouté celles de León et de Burgos. Ces dix dernières années, les Départements de Philologie Classique de ces Universités ont été chargés d'organiser des Colloques sur un thème particulier; le dixième, qui a eu lieu à León, a été consacré à la religion et mythologie classiques, et les Actes en ont été édités avec une rapidité surprenante, qui permet d'expliquer quelques errata et un saut dans l'ordre alphabétique de la bibliographie des pages 32-33. On distingue cinq groupes thématiques : 